\title{
Mercúrio em clínica odontológica: concentrações semanais no ambiente de trabalho e suas relações com procedimentos dentários em São Gonçalo, Estado do Rio de Janeiro, Brasil
}

Mercury in dental clinic: weekly concentrations in the workplace and its relations with dental procedures in São Gonçalo, Rio de Janeiro State, Brazil

Mercurio en clínica odontológica: concentraciones semanales en el ambiente de trabajo y sus relaciones con procedimientos dentales en São Gonçalo, Estado de Rio de Janeiro, Brasil

Ivisson Carneiro Medeiros da Silva

Instituto de Estudos em Saúde Coletiva, Universidade Federal do Rio de Janeiro, Rio de Janeiro, Rio de Janeiro, Brasil

Reinaldo Calixto de Campos (in memorian)

Departamento de Química, Pontifícia Universidade Católica do Rio de Janeiro, Rio de Janeiro, Rio de Janeiro, Brasil

Armando Meyer

Instituto de Estudos em Saúde Coletiva, Universidade Federal do Rio de Janeiro, Rio de Janeiro, Rio de Janeiro, Brasil
Carmen Ildes Rodrigues Froes Asmus

Instituto de Estudos em Saúde Coletiva, Universidade Federal do Rio de Janeiro, Rio de Janeiro, Rio de Janeiro, Brasil

Rachel Hauser Davis

Departamento de Química, Pontifícia Universidade Católica do Rio de Janeiro, Rio de Janeiro, Rio de Janeiro, Brasil

Volney de Magalhães Câmara

Instituto de Estudos em Saúde Coletiva, Universidade Federal do Rio de Janeiro, Rio de Janeiro, Rio de Janeiro, Brasil

\section{RESUMO}

O objetivo deste estudo foi avaliar a exposição ocupacional ao mercúrio em um consultório odontológico, através das medições das concentrações de mercúrio no ar realizadas com um analisador portátil (Lumex). As medições foram feitas a cada hora durante a semana de trabalho em um consultório, localizado em São Gonçalo, Estado do Rio de Janeiro, Brasil. Concentrações de mercúrio no ar ambiente, após a realização de 175 procedimentos, foram avaliadas. Os procedimentos foram divididos em três grupos de acordo com a utilização de mercúrio e da caneta de alta rotação. A concentração média de mercúrio observada no ar da clínica foi de $2,49 \mu \mathrm{gHg} \cdot \mathrm{m}^{-3}$, alcançando $4,69 \mu \mathrm{gHg} \cdot \mathrm{m}^{-3}$ no dia em que o maior número de procedimentos foi realizado. $O$ procedimento de colocação de amálgama levou à maior média de concentração de mercúrio no ar, mas não estatisticamente significante com relação aos outros grupos de procedimentos ( $p$-valor $=0,988)$. Os dados coletados no ambiente mostraram que as concentrações de mercúrio estavam dentro dos limites estabelecidos pela legislação brasileira e internacional.

Palavras-chave: Mercúrio; Riscos Ocupacionais; Odontologia.

\section{INTRODUÇÃO}

O mercúrio $(\mathrm{Hg})$, utilizado na odontologia para confecção de restaurações de amálgama é a causa de doenças ocupacionais em mais de 200 profissões $^{1}$. A legislação brasileira² estabelece, em 40 microgramas de $\mathrm{Hg}$ por metro cúbico de ar $\left(40 \mu \mathrm{gHg} \cdot \mathrm{m}^{-3}\right)$, o limite de exposição em ambientes de trabalho com $\mathrm{Hg}$ para uma jornada de trabalho de até $48 \mathrm{~h}$ semanais. Outras fontes não-ocupacionais devem também ser pesquisadas para uma correta avaliação da exposição de acordo com a norma ${ }^{3,4}$.

\footnotetext{
Correspondência / Correspondence / Correspondencia:

Ivisson Carneiro Medeiros da Silva

Instituto de Estudos em Saúde Coletiva/UFRJ

Av. Horácio Macedo, s/nº Ilha do Fundão. Bairro: Cidade Universitária CEP: 21941-598 Rio de Janeiro-Rio de Janeiro-Brasil

Tel.: (21) 2598-9288

E-mail: ivisson@iesc.ufri.br
}

As restaurações de amálgama utilizam $\circ \mathrm{Hg}$ em sua forma líquida que, combinado com limalha de prata, cobre e outros metais, formam uma liga utilizada como material restaurador das cavidades afetadas pela doença cárie, sendo utilizado na odontologia há mais de $150 \operatorname{anos}^{5,6}$. Na cavidade oral há evaporação do $\mathrm{Hg}$ que, após ser inalado, é absorvido pelo sangue e levado aos tecidos e órgãos diversos, podendo causar alterações, principalmente em rins e no sistema nervoso central'.

Os profissionais da área odontológica estão sujeitos à exposição ao $\mathrm{Hg}$ durante a realização de procedimentos diversos, como na remoção de restauração de amálgama deficiente, no descarte do material em excesso e na confecção de nova restauração, visto que os auxiliares também podem realizar o procedimento de colocação do material na cavidade dentária, além do contato com o spray de água-ar resultante do movimento da caneta de alta 
rotação e, ainda, por riscos de acidentes como a queda do material no consultório ${ }^{8}$. A inalação do Hg é uma das formas de exposição mais preocupantes nos casos em que houver ventilação deficiente do ambiente, falta de cumprimento das normas de manipulação do material no local de preparação e ausência de coifas na área do preparo e manejo do $\mathrm{Hg}^{9}$.

Estudos ${ }^{10,11}$ relacionaram a concentração de $\mathrm{Hg}$ em sangue, urina e cabelo com o número e tamanho das restaurações de amálgama de pacientes, obtendo significância estatística nos seus resultados em sangue e urina, porém não estatisticamente significante em relação ao cabelo.

Por sua consciência ambiental e por serem os responsáveis pelo manejo de todas as etapas da confecção das restaurações de amálgama, os profissionais da área odontológica deveriam ser conhecedores das normas de biossegurança para evitar a contaminação do meio ambiente - em respeito à legislação ambiental - e para a própria segurança e a de seus pacientes ${ }^{12,13}$

Devido à sua apolaridade, o $\mathrm{Hg}$ inalado chega à corrente sanguínea pela via respiratória e atravessa a barreira hematoencefálica, podendo causar distúrbios neurológicos. Estes efeitos foram observados em estudo sobre exposição ao $\mathrm{Hg}$ em trabalhadores de fábrica de lâmpadas do Estado de São Paulo ${ }^{14}$, Brasil, onde os autores observaram alterações na sensibilidade visual da fóvea e de regiões periféricas do campo visual. Estudos de revisão ${ }^{15}$ e de caso-controle ${ }^{16}$ evidenciaram os riscos de intoxicação ocupacional ao $\mathrm{Hg}$ e discutiram que trabalhadores expostos cronicamente podem adoecer e que o uso correto e o gerenciamento dos riscos devem ser observados. Os autores concluíram que a exposição ao vapor de $\mathrm{Hg}$ tem relação estatisticamente significante com o tempo de trabalho na atividade laboral, caracterizando a exposição ocupacional crônica ao metal.

Métodos de avaliação em campo e laboratoriais para o monitoramento do vapor de $\mathrm{Hg}$ em ambientes fechados foram comparados anteriormente ${ }^{17}$. presente trabalho tem como objetivo a avaliação da exposição ao vapor de mercúrio $\left(\mathrm{Hg}^{0}\right)$ em um consultório odontológico, por medições da sua concentração na atmosfera, buscando relações com os procedimentos odontológicos durante o ciclo de uma semana de trabalho.

\section{MATERIAIS E MÉTODOS}

Trata-se de um estudo no qual foram analisadas amostras de ar de uma clínica odontológica em uma empresa de serviço social situada no Município de São Gonçalo, Estado do Rio de Janeiro, Brasil. As medições foram realizadas durante a semana de trabalho, de modo a permitir a avaliação de toda a dinâmica das atividades relativas ao movimento de pessoal da clínica, do número de atendimentos e das variações das concentrações de $\mathrm{Hg}^{0}$. Nessa clínica trabalham 44 funcionários, sendo 20 estagiários, 12 cirurgiões-dentistas e 12 auxiliares de consultório dentário, atendendo em diversas especialidades odontológicas, como clínica geral, endodontia, prótese, periodontia e odontopediatria. A especialidade selecionada para a realização do estudo foi a clínica geral, onde se utiliza o amálgama como um dos materiais restauradores. As outras clínicas de especialidades odontológicas não utilizam amálgama rotineiramente.

Serviço de Clínica Geral apresenta seis cadeiras odontológicas ao redor de uma área de trabalho em forma hexagonal (Figura 1), na qual são realizadas as tarefas pelas auxiliares e armazenados os materiais de uso da clínica. Uma mesa central serve de suporte para o amalgamador, fotopolimerizador, luvas de procedimento e outros materiais de uso contínuo da clínica. Cada uma das seis bancadas possui uma pia com acionamento automático por movimento, além de recipientes para descarte do material utilizado e material de escritório para organização das fichas dos pacientes. Os estagiários atuam como dentistas desta clínica, sob supervisão de dois cirurgiões-dentistas da empresa. Realizam todo tratamento dos pacientes e os encaminham para as especialidades onde serão tratados por profissionais já formados e especializados em determinada área.

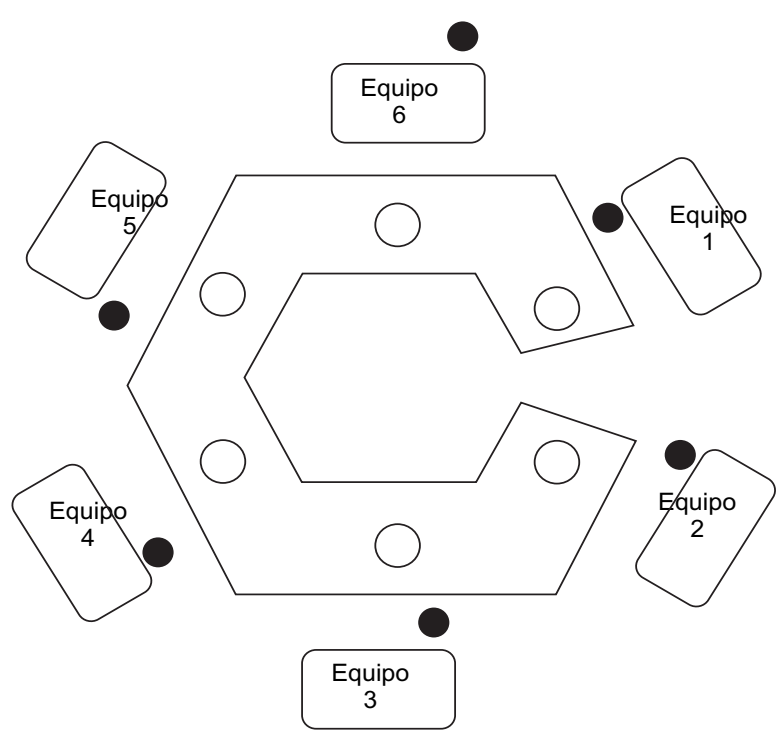

Pontos de amostragem

Figura 1 - Layout das cadeiras clínicas e dos equipamentos odontológicos. São Gonçalo, Rio de Janeiro, Brasil

A dinâmica do atendimento consiste na apresentação do paciente para consulta, que é encaminhado para a área de escovação, sob supervisão de uma auxiliar. Os estagiários levam os pacientes até a clínica, onde a auxiliar prepara o material para o procedimento que será realizado. Ao término, o estagiário assina a ficha de anamnese e o plano de tratamento do paciente com o procedimento realizado. A auxiliar entrega-lhe a carteira de agendamento da próxima consulta com o valor e o procedimento a ser pago para a próxima visita. Por turno, cada estagiário atende, em média, oito pacientes com 30 min de 
consulta agendada. No caso de falta de um paciente agendado, aqueles que estão na sala de espera têm seu horário adiantado e podem ser atendidos por outro profissional. Caso ocorra a falta de um profissional, os pacientes deste são remanejados para outros profissionais ou remarcados para outra data.

As auxiliares de consultório dentário atuam no preparo do paciente, arrumam os equipamentos, lavam o material utilizado e os encaminham para a central de esterilização, manipulam e disponibilizam o amálgama para os estagiários e fornecem materiais para os mesmos, além de também fazerem as anotações pertinentes nas fichas clínicas dos pacientes e de serem responsáveis pela organização da clínica durante o processo de trabalho.

Para a realização da análise de ar, foram amostrados seis pontos específicos da clínica, indicados na figura 1. As medições iniciaram-se na abertura da clínica, repetindo-se a cada hora subsequente, até o fechamento. A malha amostral incluiu as regiões próximas à área de atuação do estagiário, a uma altura de $1 \mathrm{~m}$ do chão aproximadamente, das seis cadeiras da clínica. Buscou-se, portanto, medir a concentração do ar que poderia ser inalado pelo participante do estudo. Para as determinações de $\mathrm{Hg}^{0}$ no ambiente de trabalho, foi utilizado o analisador de $\mathrm{Hg}$ Lumex RA-915+(R) (Saint Petersburg, Rússia), permitindo determinar as concentrações de vapor de $\mathrm{Hg}^{0}$ no ambiente de forma instantânea e contínua. Devido à sua portabilidade, as análises tornam-se bem mais simples e confiáveis, sendo possível determinar as concentrações de $\mathrm{Hg}^{0}$ nos pontos, com intervalos pequenos entre as medições, além da possibilidade de repetições a cada hora. Os valores expostos neste estudo referem-se à média das concentrações avaliadas em 1 min, em cada ponto de avaliação. $\bigcirc$ analisador Lumex RA-915+(R) apresenta um limite de detecção de $2 \mathrm{ng} / \mathrm{m}^{3}$, graças à sua célula de absorção multipasso, em conjunto com uma célula de calibração interna que permite a análise em tempo real.

Os procedimentos realizados foram divididos em três grupos, sendo: grupo I - amálgama (preparo, manipulação e aplicação do material); grupo II procedimentos avaliados que utilizavam a turbina de alta rotação (limpeza/profilaxia, polimento de restaurações e remoção de cárie); e grupo III procedimentos que não utilizavam a turbina de alta rotação (anestesia, anotação, arrumação do equipo, avaliação, curativo, exodontia, ionômero de vidro, nenhum, aplicação de resina fotopolimerizável). $\bigcirc$ procedimento definido como "nenhum" consistia no momento em que os profissionais aguardavam algum material e, por este motivo, não realizavam procedimento algum no paciente. Essa divisão foi feita tendo como base a utilização do $\mathrm{Hg}$. $\mathrm{O}$ grupo | utiliza o $\mathrm{Hg}$ diretamente. $\bigcirc$ grupo || utiliza o $\mathrm{Hg}$ indiretamente, podendo implicar em exposição ao $\mathrm{Hg}$ pela possível dissipação de metal por meio do spray de água/ar da caneta de alta/baixa rotação. $\bigcirc$ grupo III relaciona-se aos procedimentos que não utilizam a caneta de alta/baixa rotação e, consequentemente, não deveriam alterar as concentrações $\mathrm{Hg}^{0}$ no ar. Foi realizado o teste não paramétrico qui-quadrado de Pearson, categorizando as variáveis em frequências de concentrações de $\mathrm{Hg}^{0}$ menores e maiores que a mediana encontrada em cada um dos grupos.

Este estudo foi submetido ao Comitê de Ética em Pesquisa do Instituto de Estudos em Saúde Coletiva (CEP/IESC), conforme resolução 196/96 e suas complementares do Conselho Nacional de Saúde, que instituiu o sistema CEP/CONEP, sob o número 03/2009, sendo aprovado no dia 5 de agosto de 2009.

\section{RESULTADOS}

A tabela 1 mostra as concentrações de $\mathrm{Hg}^{0}$ no ar do ambiente de trabalho, no período do dia, durante uma semana.

Estes resultados evidenciaram uma grande variabilidade das concentrações de $\mathrm{Hg}^{0}$ no ambiente durante a semana de trabalho. As médias mínimas e máximas em cada hora de avaliação, observadas durante a semana de trabalho, variaram de 0,39 (DP - 0,09) a 7,43 (DP - 2,39) $\mu \mathrm{gHg} \cdot \mathrm{m}^{-3}$, com média geral de $2,49 \mu \mathrm{gHg} \cdot \mathrm{m}^{-3}$ (DP - 0,69).

Tabela 1 - Média (e desvio padrão) das concentrações de mercúrio $\left(\mu \mathrm{gHg} \cdot \mathrm{m}^{-3}\right.$ ) no ar durante a semana de estudo na clínica odontológica. São Gonçalo, Rio de Janeiro, Brasil

\begin{tabular}{|c|c|c|c|c|c|c|c|c|c|c|}
\hline \multicolumn{11}{|c|}{ Horário da medição (h) } \\
\hline 08:00 & 09:00 & $10: 00$ & $11: 00$ & $12: 00$ & 13:00 & $14: 00$ & $15: 00$ & $16: 00$ & $17: 00$ & Média \\
\hline
\end{tabular}

Dia $1 \quad 1,84(0,18) 3,33(0,10) 3,16(0,27) 2,93(0,14) \quad 1,45(0,53) \quad 1,05(0,43) \quad 1,63(0,19) \quad 1,48(0,21) 3,52(0,82) 5,31(0,82) 2,57(1,32)$

Dia $2 \quad 2,44(0,46) \quad 2,3(0,27) \quad 3,10(0,50) 2,51(0,33) \quad 1,38(0,92) \quad 0,94(0,19) \quad 1,89(0,32) 2,51 \quad(0,84) 2,36(0,42) \quad 1,62(0,58) 2,10(0,64)$

Dia $32,60(0,45) 7,43(2,39) \quad 7,22(2,14) 7,10(0,77) 6,41(1,17) 2,74(1,80) 3,67(1,35) 4,00(0,95) 3,24(0,25) 2,45(0,76) 4,69(2,10)$

Dia $4 \quad 6,00(1,93) 3,31(0,69) 2,06(0,40) 3,38(0,21) 2,74(0,44) \quad 1,53(0,58) \quad 1,14(1,30) 0,59(0,20) 0,73(0,07) \quad 1,44(0,62) 2,29(1,64)$

Dia $50,63(0,11) 0,74(0,75) 0,41(0,11) 0,39(0,09) 0,42(0,07) 0,51(0,05) 0,74(0,12) 0,87(0,18) 0,83(0,16) 2,34(0,68) 0,79(0,57)$

Média $2,70(0,5) \quad 3,42(0,17) \quad 3,19(0,42) 3,26(0,09) 2,48(0,49) \quad 1,35(0,53) \quad 1,81(0,54) \quad 1,89(0,33) 2,14(0,22) \quad 2,63(0,28) 2,49(0,69)$ 
Foram medidas as concentrações de $\mathrm{Hg}^{0}$ no ar relativas a 175 procedimentos, durante a semana de trabalho. A tabela 2 mostra a frequência de procedimentos por dia de serviço e as concentrações de $\mathrm{Hg}^{0}$ no ambiente no momento da realização destes procedimentos. A tabela 3 apresenta as médias de $\mathrm{Hg}^{0}$ $\left(\mu \mathrm{g} / \mathrm{m}^{3}\right)$ associadas às diferentes intervenções clínicas avaliadas, divididas nos três grupos, segundo o uso de $\mathrm{Hg}^{0}$, ao longo da semana de trabalho.

Realizando a comparação entre os três grupos de procedimentos, observa-se uma maior média de concentração de $\mathrm{Hg}^{0}$ no grupo I, sendo seguido pelo grupo de procedimentos que utilizam caneta de alta rotação (grupo II) e, por último, os outros procedimentos que não utilizam caneta de alta rotação (grupo III). $O$ teste não paramétrico qui-quadrado de
Pearson, avaliando a frequência de eventos menores e maiores que a mediana da concentração de $\mathrm{Hg}^{0}$ em cada grupo, obteve um $p$-valor $=0,988$, resultado não significante estatisticamente.

Devido à disposição das cadeiras da clínica e da falta de barreiras físicas entre os equipamentos, não se pode afastar a possibilidade de ocorrer variação da concentração de $\mathrm{Hg}^{0}$ no ar nos pontos de coleta de amostras que seriam provenientes de cadeiras adjacentes ou devido à emissão de $\mathrm{Hg}$ em procedimentos anteriores. A figura 2 mostra as médias das concentrações de $\mathrm{Hg}^{0}$ observadas nos cinco dias em cada cadeira odontológica, mostrando que as variações observadas a cada hora, ao longo da semana, mostram padrões bastante similares em todos os equipamentos.

Tabela 2 - Número de procedimentos odontológicos avaliados por dia de trabalho e a média das concentrações de mercúrio ( $\left.\mu \mathrm{gHg} \cdot \mathrm{m}^{-3}\right)$, São Gonçalo, Rio de Janeiro, Brasil

\begin{tabular}{|c|c|c|c|c|c|c|c|c|c|c|c|c|}
\hline \multirow{2}{*}{ Procedimento } & \multicolumn{12}{|c|}{ Número de procedimentos, média e desvio padrão } \\
\hline & & Dia 1 & & Dia 2 & & Dia 3 & & Dia 4 & & Dia 5 & & Total \\
\hline Amálgama & 3 & $2,75(0,70)$ & 3 & $2,14(0,90)$ & 3 & $6,77(2,20)$ & 4 & $3,57(2,80)$ & 2 & $0,69(0,02)$ & 15 & $3,38(2,60)$ \\
\hline Anestesia & 1 & 1,64 & 3 & $2,65(0,66)$ & 2 & $2,47(0,53)$ & - & - & - & - & 6 & $2,42(0,60)$ \\
\hline Anotações & 3 & $2,61(1,16)$ & 6 & $2,11(0,68)$ & 6 & $5,33(1,68)$ & 8 & $2,11(2,05)$ & 6 & $0,68(0,06)$ & 29 & $2,53(1,79)$ \\
\hline $\begin{array}{l}\text { Organização do } \\
\text { equipamento }\end{array}$ & 3 & $2,54(1,30)$ & 5 & $1,97(1,52)$ & 3 & $4,42(1,50)$ & 5 & $2,09(1,21)$ & 1 & 0,99 & 17 & $2,48(1,47)$ \\
\hline Avaliação & 5 & $1,86(0,84)$ & 5 & $2,65(1,45)$ & 2 & $5,61(1,20)$ & 4 & $3,27(1,54)$ & 1 & 0,78 & 17 & $2,80(1,81)$ \\
\hline Curativos & - & - & 2 & $2,34(0,52)$ & 2 & $5,74(1,05)$ & - & - & 3 & $0,66(0,10)$ & 7 & $2,59(1,54)$ \\
\hline Exodontia & 1 & 1,36 & 1 & 3,23 & - & - & 1 & 0,62 & - & - & 3 & $1,73(1,34)$ \\
\hline lonômero de vidro & 1 & 3,09 & 3 & $2,13(0,65)$ & 2 & $4,31 \quad(0,80)$ & 1 & 0,68 & 2 & $0,52(0,05)$ & 9 & $2,20(1,48)$ \\
\hline Profilaxia/limpeza & 3 & $2,59(1,08)$ & - & - & 1 & 8,48 & 1 & 6,41 & 1 & 0,57 & 6 & $3,87(2,94)$ \\
\hline Nenhum & 3 & $2,20(1,24)$ & 6 & $2,22(0,92)$ & 6 & $4,30(1,51)$ & 6 & $1,84(1,30)$ & 7 & $0,61(0,12)$ & 28 & $2,18(1,54)$ \\
\hline Polimento & 2 & $3,25(1,32)$ & 1 & 0,76 & 1 & 2,58 & 1 & 1,43 & 1 & 0,47 & 6 & $1,96(1,23)$ \\
\hline Remoção de cárie & 7 & $2,45(1,58)$ & 3 & $2,38(0,54)$ & 6 & $5,53(2,54)$ & 4 & $1,06(0,59)$ & 1 & 0,98 & 21 & $2,98(2,45)$ \\
\hline $\begin{array}{l}\text { Resina } \\
\text { fotopolimerizável }\end{array}$ & 4 & $2,35(1,20)$ & 4 & $2,75(1,34)$ & 3 & $5,85(1,48)$ & - & - & - & - & 11 & $3,45(2,65)$ \\
\hline $\begin{array}{l}\text { Total de } \\
\text { procedimentos }\end{array}$ & 36 & & 42 & & 37 & & 35 & & 25 & & 175 & \\
\hline
\end{tabular}

Sinal convencional utilizado: - Dado numérico igual a zero não resultante de arredondamento.

Tabela 3 - Média das concentrações de mercúrio $\left(\mu \mathrm{gHg} \cdot \mathrm{m}^{-3}\right)$ no ar por grupos de procedimentos e dias da semana e desvio padrão. São Gonçalo, Rio de Janeiro, Brasil

\begin{tabular}{ccccccc}
\hline & Dia 1 & Dia 2 & Dia 3 & Dia 4 & Dia 5 & Média \\
\hline Grupo 1 & $2,75(0,70)$ & $2,14(0,90)$ & $6,77(2,20)$ & $3,57(2,80)$ & $0,69(0,02)$ & $3,38(2,60)$ \\
Grupo 2 & $2,62(0,82)$ & $1,97(0,68)$ & $5,53(2,34)$ & $2,02(0,96)$ & $0,67(0,24)$ & $2,96(0,79)$ \\
Grupo 3 & $2,23(1,50)$ & $2,35(0,85)$ & $4,80(2,36)$ & $2,11(1,77)$ & $0,66(0,74)$ & $2,52(0,67)$ \\
\hline
\end{tabular}




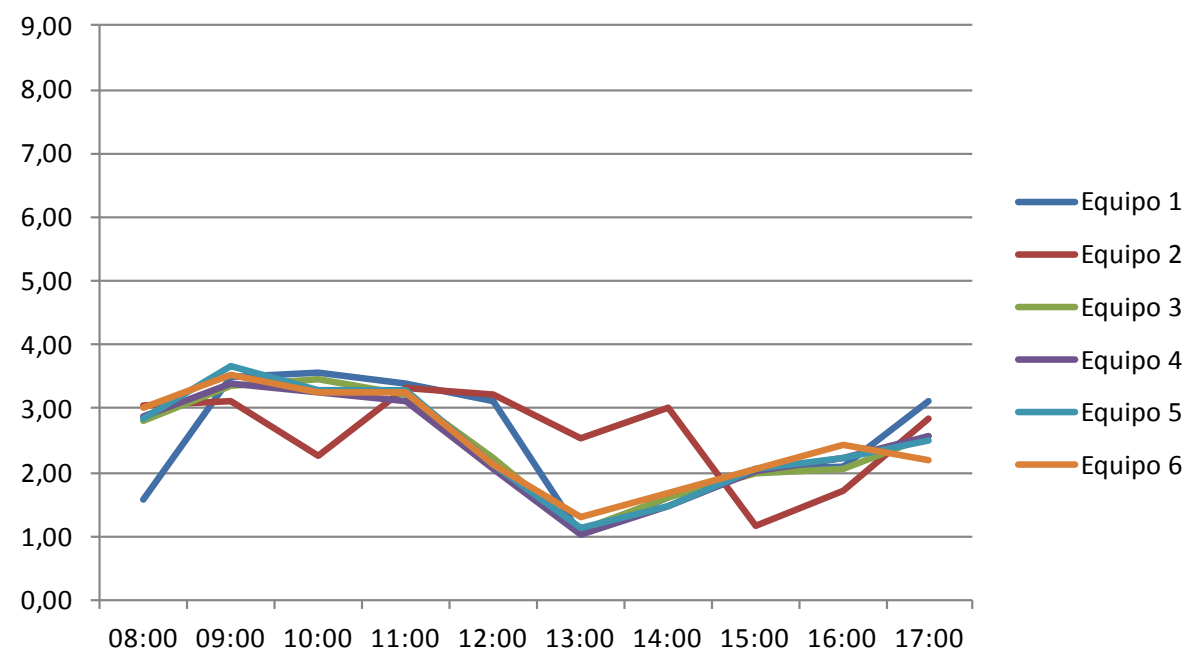

Figura 2 - Média das concentrações de mercúrio $\left(\mu \mathrm{gHg} \cdot \mathrm{m}^{-3}\right)$ no ar por hora de medição e por equipo durante o período de estudo. São Gonçalo, Rio de Janeiro, Brasil

\section{DISCUSSÃO}

Os resultados avaliados na tabela 1 não são superiores aos estabelecidos pela legislação trabalhista brasileira ${ }^{2}$, que é de $40 \mu \mathrm{gHg} \cdot \mathrm{m}^{-3}$, nem aos estabelecidos por outros órgãos internacionais como - Centro de Controle e Prevenção de Doenças dos Estados Unidos $(C D C)^{18}$, que estabelece a concentração de $50 \mu \mathrm{gHg} \cdot \mathrm{m}^{-3}$, e estão próximos aos estudos de exposição ao $\mathrm{Hg}$ ocupacional em trabalhadores da área odontológica na Suécia, com achados médios entre 1,8 e 2,1 $\mu \mathrm{gHg} \cdot \mathrm{m}^{-3}$ no ambiente com seis clínicas odontológicas ${ }^{19}$. O limite máximo de exposição permitida (PEL) para $\circ \mathrm{Hg}$ pela Occupational Safety \& Health Administration (OSHA) é de $100 \mu \mathrm{g} / \mathrm{m}^{3}$ de ar. Este valor não pode ser excedido em nenhum momento no ambiente de trabalho. Os resultados deste estudo determinaram valores bem abaixo desse referencial ${ }^{20}$.

A média da concentração diária de $\mathrm{Hg}^{0}$ no ar do ambiente da clínica variou de 0,79 (DP - 0,57) e 4,69 (DP $-2,10) \mu \mathrm{g} / \mathrm{m}^{3}$. O terceiro dia apresentou a maior média de $\mathrm{Hg}^{0}$ no ar, quando também foi relatado um aumento da temperatura ambiente pelos participantes do estudo, o que poderia estar relacionado a esta maior média ${ }^{21,22}$. $\bigcirc$ procedimento amálgama (grupo I) apresentou média de $3,38(\mathrm{DP}-2,50) \mu \mathrm{g} / \mathrm{m}^{3}$ neste tipo de procedimento, com médias diárias variando de 0,69 (DP - 0,71) a 6,77 (DP - 1,92) $\mu \mathrm{g} / \mathrm{m}^{3}$ de ar. Outros estudos $^{21,23}$ mostraram que, durante a realização de procedimentos com o amálgama, a concentração de $\mathrm{Hg}^{0}$ variou de 1,0 a 8,0 e de 1,8 a $2,1 \mu \mathrm{g} / \mathrm{m}^{3}$.

O último dia analisado apresentou a menor concentração de $\mathrm{Hg}^{0}$ no ambiente de trabalho, possivelmente por ter sido realizado o menor número de procedimentos da semana ( 25 procedimentos) e pelo menor número de trabalhadores presentes. Nos outros dias, variações térmicas não foram relatadas, nem a falta de pessoal foi observada e as concentrações de $\mathrm{Hg}^{0}$ no ambiente variaram pouco, de 2,10 a $2,57 \mu \mathrm{g} / \mathrm{m}^{3}$ e a quantidade de procedimentos variou de 35 a 42 nestes dias.

\section{CONCLUSÃO}

As concentrações de $\mathrm{Hg}^{0}$ no ar do ambiente de trabalho mostraram-se, ao longo de todo o estudo e em todas as medidas, abaixo dos limites estabelecidos pela legislação brasileira e por outros órgãos internacionais. Em relação aos procedimentos, observou-se que o manuseio da cápsula no amalgamador e a introdução do amálgama na cavidade dentária foram responsáveis pela maior média das concentrações de $\mathrm{Hg}^{0}$ no ar do ambiente de trabalho, em concordância com os resultados observados na literatura.

Os estagiários que atuavam como dentistas na clínica utilizavam corretamente os equipamentos individuais de biossegurança, em contraste com o que foi observado nas auxiliares, que não utilizavam as máscaras de proteção em todo o período que se encontravam no interior da clínica. Apesar dos valores encontrados de $\mathrm{Hg}^{0}$ no ar estarem dentro das normas brasileiras e internacionais, os equipamentos de proteção devem ser utilizados de modo a minimizar os riscos de exposição e efeitos causados pelo $\mathrm{Hg}^{0}$.

A temperatura no terceiro dia de avaliação pode ter contribuído para um aumento da concentração do $\mathrm{Hg}^{0}$ no ar neste dia, embora medições da temperatura ambiente não estivessem incluídas no desenho do estudo, não sendo possível, portanto, uma conclusão sobre o efeito desta variável. Os dentistas supervisores não foram avaliados no presente estudo porque não trabalhavam diretamente no atendimento da clínica geral, de modo que alguns profissionais atendiam em outras especialidades e não estariam no local de avaliação.

\section{AGRADECIMENTOS}

Ao Laboratório de Estudos Ambientais, do Departamento de Química da Pontifícia Universidade Católica do Rio de Janeiro, pelas análises e interpretações dos dados ambientais. 


\title{
Mercury in dental clinic: weekly concentrations in the workplace and its relations with dental procedures in São Gonçalo, Rio de Janeiro State, Brazil
}

\begin{abstract}
The aim of this study was to evaluate occupational exposure to mercury in a dental office, measuring mercury concentrations by using a portable mercury analyzer (Lumex). Measurements were taken every hour during the working week in an office, located in São Gonçalo, Rio de Janeiro State, Brazil. Mercury concentrations in ambient air were evaluated after performing 175 procedures. The procedures were divided in three groups according to the use of mercury and the dental high-speed handpiece. The average mercury concentration observed in the clinic's air was $2.49 \mu \mathrm{gHg} \cdot \mathrm{m}^{-3}$, reaching $4.69 \mu \mathrm{gHg} \cdot \mathrm{m}^{-3}$ on the day on which the greatest number of procedures was conducted. The procedure of placing amalgam led to the highest average of mercury concentration in the air, but it was not statistically significant in relation to other groups of procedures ( $p$-value $=0.988)$. The data collected in the environment have shown that mercury concentrations were within the limits established by Brazilian and international law.
\end{abstract}

Keywords: Mercury; Occupational Risks; Dentistry.

\section{Mercurio en clínica odontológica: concentraciones semanales en el ambiente de trabajo y sus relaciones con procedimientos dentales en São Gonçalo, Estado de Rio de Janeiro, Brasil}

\section{RESUMEN}

El objetivo de este estudio fue el de evaluar la exposición ocupacional al mercurio en un consultorio odontológico, a través de las mediciones de las concentraciones de mercurio en el aire realizadas con un analizador portátil (Lumex). Las mediciones se hicieron a cada hora durante la semana de trabajo en un consultorio, localizado en São Gonçalo, Estado de Rio de Janeiro, Brasil. Fueron evaluadas las concentraciones de mercurio en el aire del ambiente, luego de la realización de 175 procedimientos. Los procedimientos se dividieron en tres grupos de acuerdo a la utilización de mercurio y de la lapicera de alta rotación. El promedio de concentración de mercurio observada en el aire de la clínica fue de 2,49 $\mu \mathrm{gHg} \cdot \mathrm{m}^{-3}$, alcanzando $4,69 \mu \mathrm{gHg} \cdot \mathrm{m}^{-3}$ el día en que se realizó el mayor número de procedimientos. El procedimiento de colocar amalgama condujo al mayor promedio de concentración de mercurio en el aire, aunque no estadísticamente significante con relación a los otros grupos de procedimientos $(p$-valor $=0,988)$. Los datos recolectados en el ambiente mostraron que las concentraciones de mercurio estaban dentro de los límites establecidos por la legislación brasileña e internacional.

Palabras clave: Mercurio; Riesgos Laborales; Odontología.

\section{REFERÊNCIAS}

1 Ministério da Saúde (BR). Agência Nacional de Vigilância Sanitária. Manual de biossegurança em odontologia; 2001.

2 Ministério do Trabalho e Emprego (BR). Secretaria de Inspeção do Trabalho. Portaria n ${ }^{\circ}$ 3.214, de 8 de junho de 1978. Aprovam as Normas RegulamentadorasNR-do Capítulo V, Título II, da Consolidação das Leis do Trabalho, relativas à Segurança e Medicina do Trabalho. Norma regulamentadora 15. Atividades e operações insalubres. Diário Oficial da União, Brasília, supl. 6 jul 1978.

3 Wrbitzky R. Unusual non-occupational exposure to metals. Bioinorg Chem Appl. 2003;1 (1):45-51.

4 Glina DMR, Satut BTG, Andrade EMOAC. A exposição ocupacional ao mercúrio metálico no módulo odontológico de uma unidade básica de saúde localizada na cidade de São Paulo. Cad Saude Publica. 1997 abr;13(2):257-67.
5 American Dental Association. Amalgam fillings. Chicago; 2008.

6 World Health Organization. Inorganic mercury. Geneva: World Health Organization; 1991. (Environmental Health Criteria; 1 18).

7 Maserejian NN, Trachtenberg FL, Assmann SF, Barregard L. Dental amalgam exposure and urinary mercury levels in children: the New England children's amalgam trial. Environ Health Perspect. 2008 Feb; 1 16(2):256-62.

8 Mason HJ, Hindell P, Williams NR. Biological monitoring and exposure to mercury. Occup Med (Lond). 2001 Feb;51(1):2-11.

9 Câmara VM, Campos RC, Frankel P, Perez MA, 10 Falcão MP. Estudo comparativo dos efeitos da utilização de mercúrio por dentistas. Cad Saude Publica. 1990 abr-jun;6(2):186-200. 
10 Hansen G, Victor R, Engeldinger E, Schweitzer C. Evaluation of the mercury exposure of dental amalgam patients by the mercury triple test. Occup Environ Med. 2004 Jun;61(6):535-40.

11 Kingman A, Albertini T, Brown LJ. Mercury concentrations in urine and whole blood associated with amalgam exposure in a US military population. J Dent Res. 1998 Mar;77(3):461-71.

12 Presidência da República (BR). Lei nº 11.889, de 24 de dezembro de 2008. Regulamenta o exercício das profissões de Técnico em Saúde Bucal-TSB e de Auxiliar em Saúde Bucal-ASB. Diário Oficial da União, Brasília, p. 2, 26 dez 2008. Seção 1.

13 Hiltz M. The environmental impact of dentistry. J Can Dent Asso. 2007 Feb;73(1):59-62.

14 Barboni MTS, Costa MF, Moura ALA, Feitosa-Santana C, Gualtieri M, Lago $M$, et al. Visual field losses in workers exposed to mercury vapor. Environ Res. 2008 May; 107(1):124-31.

15 Grigoletto JC, Oliveira AS, Muñoz SIS, Alberguini LBA, Takayanagui AMM. Exposição ocupacional por uso de mercúrio em odontologia: uma revisão bibliográfica. Cienc Saude Coletiva. 2008 marabr;13(2):533-42.

16 Trzcinka-Ochocka M, Gazewski A, Brodzka R. Exposure to mercury vapors in dental workers in Poland. Int J Occup Med Environ Health. 2007;20(2): 147-53.
17 Singhvi R, Turpin R, Kalnicky DJ, Patel J. Comparison of field and laboratory methods for monitoring metallic mercury vapor in indoor air. J Hazard Mater. 2001 May;83(1-2):1-10.

18 United States. Department of Health and Human Services. Centers for Disease Control and Prevention. National Institute for Occupational Safety and Health. Niosh pocket guide to chemical hazards; 2007.

19 Langworth S, Sallsten G, Barregard L, Cynkier I, Lind $\mathrm{ML}$, Soderman E. Exposure to mercury vapor and impact on health in the dental profession in Sweden. J Dent Res. 1997 Jul;76(7):1397-404.

20 United States. Department of Labor. Occupational Safety e Health Administration. [Internet] [cited Aug 2009]. Available from: http://www.osha.gov/SLTC/ healthguidelines/mercuryvapor/recognition.html.

21 United States. Department of Health and Human Services. Public Health Service. Agency for Toxic Substances and Disease Registry. Toxicological profile for mercury. Atlanta; 1999.

22 World Health Organization. Elemental mercury and inorganic mercury compounds: human health aspects. Geneva: World Health Organization; 2003.

23 Engle JH, Ferracane JL, Wichmann J, Okabe T. Quantitation of total mercury vapor released during dental procedures. Dent Mater. 1992 May;8(3):17680. 